\section{(1) \\ CrossMark}

\title{
Sarcoidosis and aspergillosis: a tough combination
}

\author{
David W. Denning
}

Affiliation: The National Aspergillosis Centre, University Hospital of South Manchester, University of Manchester, Academic Health Science Centre, Manchester, UK.

Correspondence: David W. Denning, The National Aspergillosis Centre, University Hospital of South Manchester, Southmoor Road, Manchester, M23 9LT, UK. E-mail: ddenningamanchester.ac.uk

@ERSpublications

CPA is a serious complication of sarcoidosis contributing to fibrosis, respiratory failure and haemoptysis http://ow.ly/cMyQ30bNG4z

Cite this article as: Denning DW. Sarcoidosis and aspergillosis: a tough combination. Eur Respir J 2017; 49: 1700574 [https://doi.org/10.1183/13993003.00574-2017].

It should have been a wake-up call. Thirty years ago, TombInson and SAHN [1] found that seven out of 12 (58\%) patients with aspergillosis complicating sarcoidosis died over a 2 year period. Three years earlier, Wollschlager and Khan [2] had found evidence of aspergillosis in 12\% of 100 consecutive sarcoidosis referrals, and aspergillomas were found in $53 \%$ of the 19 patients with fibrocystic aspergillosis. Yet the literature was almost silent on this topic despite an estimated global prevalence of 1.2 million patients with sarcoidosis [3], until 2008. In that year, Hours et al. [4] surveyed pulmonary cavitary sarcoidosis and its complications in their tertiary care service for sarcoidosis in Paris, France. They found that 3.9\% had cavitary lesions and $2.1 \%$ an aspergilloma. This team have followed that report up in this issue of the European Respiratory Journal with a detailed study of chronic pulmonary aspergillosis complicating pulmonary sarcoidosis [5]. Others have noted the complexity of this dual pulmonary condition [6].

Sarcoidosis remains an enigmatic disorder of uncertain aetiology. Early reports focused on the cutaneous manifestations, and it was Cæsar Boeck in Oslo who emphasised the multisystem nature of the disease in 1916, including pulmonary disease [7]. The Kveim test was introduced in 1941. Wurm, Reindell and Heilmeyer demonstrated the benefits of a radiographic staging system for sarcoidosis, which was adapted and expanded by others, including Citron, Siltzbach, Chrétien and DeRemeE [8]. Aspergilloma complicates stage III or fibrocystic/cavitary pulmonary sarcoidosis. Predicting which patients with sarcoidosis will develop fibrosis or cavitation is currently not possible, just as the prediction of who will develop aspergillosis is equally obscure.

Chronic pulmonary aspergillosis (CPA) comprises four entities: chronic cavitary (CCPA), simple aspergilloma, Aspergillus nodule and - the most severe - chronic fibrosing (CFPA) or destroyed lung [9]. In the context of cavitary sarcoidosis, defining CCPA is difficult because cavitary sarcoidosis is similar in appearance to CCPA, until a fungal ball is visible. UZUNHAN et al. [5] have, for the first time, defined the different CPA phenotypes in sarcoidosis based on imaging and Aspergilllus IgG serology, with additional data on culture and also documented outcomes. In a 25 year cohort of 3137 sarcoidosis patients, 80 were identified with CPA (2.6\%) and 65 were analysed in detail. An aspergilloma was seen in 41 (68\%) patients, 60 (92\%) had positive Aspergillus IgG serology and 48 (81\%) grew Aspergillus spp. from a respiratory sample. Only four (7\%) were Aspergillus IgG serology-only positive using multiple tests evolved over the 25 years of their study. Nine out of 34 (26\%) patients had detectable Aspergillus antigen

Received: March 192017 | Accepted: March 212017

Conflict of interest: Disclosures can be found alongside this article at erj.ersjournals.com

Copyright CERS 2017 
in the blood. CPA was present at the diagnosis of sarcoidosis in $10(15 \%)$ of cases and developed over time in the remainder.

In most patients, CPA was classifiable - simple aspergilloma was seen in 17 (31\%), CCPA in 27 (49\%) and a mixed pattern including CFPA in 8 (15\%) [5]. Compared with other underlying disease entities, this is a higher proportion of simple aspergilloma and CFPA. Aspergillus nodules are found in fewer than $10 \%$ of patients with CPA [10], and, in sarcoidosis, nodules are uncommon as the main radiological presentation [11].

Sarcoidosis is more common in black people living in the USA and London. Elsewhere, whether African heritage is a risk factor for sarcoidosis is not known. In this large French cohort, a substantial proportion of those with cavitary sarcoidosis (39\%) [4] and with CPA complicating sarcoidosis (34\%) were black [5]. It is not clear if black people are more or less likely to develop CPA in sarcoidosis than others.

There is a link between environmental fungal exposure and the development of sarcoidosis [12, 13] and some occupational exposures to fungi were quite common in those with CPA and sarcoidosis [5]. The overall importance of these associations has not been clarified, but could be relevant in the context of CPA as antifungal therapy is usually given. UzunHAN et al. [5] treated $85 \%$ of their patients with antifungal therapy for at least 6 weeks. Cough and the general symptoms of weight loss and fatigue improved in most patients.

Some patients with sarcoidosis are mildly immunocompromised, as evidenced by low CD4 cell counts and purified protein derivative anergy $[14,15]$, irrespective of corticosteroid therapy. We have found that many patients with CPA have reduced natural killer cells and gamma interferon and/or interleukin (IL)-12 production [16, 17]. Further immunological investigations of those with cavitary sarcoidosis and CPA complicating sarcoidosis are called for, as immunotherapy (i.e. gamma interferon replacement) may be beneficial.

UzUNHAN et al. [5] report surgical resection in 11 patients, usually for haemoptysis. Despite this and 3 months of antifungal therapy after surgery, 10 (91\%) relapsed, 6 to 45 months after surgery. This is a much higher relapse rate than for CPA overall, in which a $25 \%$ relapse rate has been documented [18]. A surgical solution for CPA in sarcoidosis should be one of last resort.

In their centre, the overall survival for CPA in sarcoidosis did not differ in this series from those with cavitary sarcoidosis $-74 \%$ at 5 years and $61 \%$ at 10 years [5]. These survival rates are much better than reported by TOMLINSON and SAHN [1], but the earlier poor outcome could reflect corticosteroid use, which accelerates the progression of CPA in the absence of effective antifungal therapy. Our series of nearly 400 patients with CPA, just published, found that sarcoidosis patients with CPA had an outcome similar to other underlying diseases [19] and not as good overall as the French series (62\% survival at 5 years, $47 \%$ at 10 years). Patients with CPA complicating non-tuberculous mycobacterial infection and chronic obstructive pulmonary disease fared worse overall [19].

The presence of a fungal ball or aspergilloma is a late feature of CPA. The presence of a fungal ball in the context of CCPA is a poor prognostic feature [19], especially if bilateral. Furthermore, antifungal resistance is more likely to develop in patients with higher fungal loads [20]. Mutations occur frequently in aspergillomas over time [21]. Given the difficulty in diagnosing CPA in sarcoidosis patients with cavitary disease, there is a case for proleptically screening patients for the development of Aspergillus IgG antibody to pick up sero-conversion before aspergillomas are visible radiologically. Earlier treatment could avoid haemoptysis, the development of general symptoms of CPA, and might improve outcome, as less fibrosis and development of CFPA is likely to occur with good antifungal therapy. This strategy needs prospective study.

Many genetic associations have now been made between CPA and certain immunological variants, namely the IL-1 pathway, IL-15, IL-10, vascular endothelial growth factor A (VEGF-A), transforming growth factor $\beta 1$ (TGF- $\beta 1$ ), DENND1B and PLAT [22-24]. In sarcoidosis, genetic variants in several pathways and genes have been found, including the IL-23/T helper (Th) 17 signalling pathway [25], butyrophilin-like 2 (BTNL2) [26], and annexin A11 (ANXA11) [27], although none is very strong. No genetic association work has yet addressed the different pulmonary phenotypes of sarcoidosis [28, 29]. If the CPA variants found in all populations are also found in sarcoidosis, then risk assessment using genetic testing might be a simpler and more comprehensive approach to excluding CPA risk, with a focus on those with genetic risks for CPA development.

CPA is one of the serious complications of sarcoidosis, partly because it contributes to fibrosis and respiratory failure and partly because of life-threatening and fatal haemoptysis. It also contributes to a worse quality of life. With an estimated annual incidence of 20640 CPA cases and a prevalence of $\sim 72000$ 
CPA patients with sarcoidosis [3], addressing the uncertainties in diagnosis and management of CPA is called for. UzunHan et al. [5] provide an excellent basis for planning future studies.

\section{References}

Tomlinson JR, Sahn SA. Aspergilloma in sarcoid and tuberculosis. Chest 1987; 92: 505-508.

2 Wollschlager C, Khan F. Aspergillomas complicating sarcoidosis. A prospective study in 100 patients. Chest 1984; 86: 585-588.

3 Denning DW, Pleuvry A, Cole DC. Global burden of chronic pulmonary aspergillosis complicating sarcoidosis. Eur Respir J 2013; 41: 621-626.

4 Hours S, Nunes H, Kambouchner M, et al. Pulmonary cavitary sarcoidosis: clinico-radiologic characteristics and natural history of a rare form of sarcoidosis. Medicine (Baltimore) 2008; 87: 142-151.

5 Uzunhan Y, Nunes H, Jeny F, et al. Chronic pulmonary aspergillosis complicating sarcoidosis. Eur Respir J 2017; 49: 1602396.

6 Pena TA, Soubani AO, Samavati L. Aspergillus lung disease in patients with sarcoidosis: a case series and review of the literature. Lung 2011; 189: 167-172.

7 Danbolt N. The historical aspects of sarcoidosis. Postgrad Med J 1958; 34: 245-247.

8 DeRemee RA. The roentgenographic staging of sarcoidosis. Historic and contemporary perspectives. Chest 1983; 83: $128-133$.

9 Denning DW, Cadranel J, Beigelman-Aubry C, et al. Chronic pulmonary aspergillosis - rationale and clinical guidelines for diagnosis and management. Eur Respir J 2016; 47: 45-68.

10 Muldoon EG, Sharman A, Page ID, et al. Aspergillus nodules; another presentation of chronic pulmonary aspergillosis. BMC Pulm Med 2016; 16: 123.

11 Malaisamy S, Dalal B, Bimenyuy C, et al. The clinical and radiologic features of nodular pulmonary sarcoidosis. Lung 2009; 187: 9-15.

12 Rossman MD, Kreider ME. Lesson learned from ACCESS (a case controlled etiologic study of sarcoidosis). Proc Am Thorac Soc 2007; 4: 453-456.

13 Terčelj M, Salobir B, Harlander M, et al. Fungal exposure in homes of patients with sarcoidosis - an environmental exposure study. Environ Health 2011; 10: 8.

14 Lee NS, Barber L, Kanchwala A, et al. Low levels of NF- $\mathrm{kB} / \mathrm{p} 65$ mark anergic CD4+ T cells and correlate with disease severity in sarcoidosis. Clin Vaccine Immunol 2011; 18: 223-234.

15 Oswald-Richter KA, Richmond BW, Braun NA, et al. Reversal of global CD4+ subset dysfunction is associated with spontaneous clinical resolution of pulmonary sarcoidosis. J Immunol 2013; 190: 5446-5453.

16 Doffinger R, Harris C, Lear S, et al. Impaired Th1 and Th17 Immunity in Chronic Pulmonary Aspergillosis. 6th Advances against Aspergillosis (6th AAA) conference, 2014, Madrid, Spain. Available from www. advancesagainstaspergillosis.org/2014/index.htm

17 Bongomin F, Harris C, Foden P, et al. Innate and adaptive immune defects in chronic pulmonary aspergillosis. $J$ Fungi 2017; in press.

18 Farid S, Mohammed S, Devbhandari M, et al. Results of surgery for chronic pulmonary aspergillosis, optimal antifungal therapy and proposed high risk factors for recurrence - a National Centre's experience. J Cardiothorac Surg 2013; 8: 180 .

19 Lowes D, Al-Shair K, Newton PJ, et al. Predictors of mortality in chronic pulmonary aspergillosis. Eur Respir J 2017; 49: 1601062.

20 Howard SJ, Cesar D, Anderson MJ, et al. Frequency and evolution of azole resistance in Aspergillus fumigatus associated with treatment failure. Emerg Infect Dis 2009; 15: 1068-1076.

21 Howard SJ, Pasqualotto A, Anderson MJ, et al. Major variations in Aspergillus fumigatus arising within aspergillomas in chronic pulmonary aspergillosis. Mycoses 2013; 56: 434-441.

22 Sambatakou H, Pravica V, Hutchinson I, et al. Cytokine profiling of pulmonary aspergillosis. Int J Immunogenetics 2006; 33: 297-302.

23 Smith NL, Bowyer P, Simpson A, et al. A prominent role for IL1 pathway and IL15 in susceptibility to chronic cavitary pulmonary aspergillosis. Clin Microbiol Infect 2014; 20: O480-O488.

24 Smith NS, Bowyer P, Simpson A, et al. Reduced expression of TLR3, TLR10 and TREM1 by human macrophages in chronic cavitary pulmonary aspergillosis, and novel associations of VEGFA, DENND1B and PLAT. Clin Microbiol Infect 2014; 20: O960-O968.

25 Fischer A, Ellinghaus D, Nutsua $\mathrm{M}$, et al. Identification of immune-relevant factors conferring sarcoidosis genetic risk. Am J Respir Crit Care Med 2015; 192: 727-736.

26 Lin Y, Wei J, Fan L, et al. BTNL2 gene polymorphism and sarcoidosis susceptibility: a meta-analysis. PLoS One 2015; 10: e0122639.

27 Feng X, Zang S, Yang Y, et al. Annexin A11 (ANXA11) gene polymorphisms are associated with sarcoidosis in a Han Chinese population: a case-control study. BMJ Open 2014; 4: e004466.

28 Judson MA. Strategies for identifying pulmonary sarcoidosis patients at risk for severe or chronic disease. Exp Rev Respir Med 2017; 11: 111-118.

29 Jeny F, Bouvry D, Freynet O, et al. Management of sarcoidosis in clinical practice. Eur Respir Rev 2016; 25: $141-150$. 